Portland State University

PDXScholar

Mathematics and Statistics Faculty

Fariborz Maseeh Department of Mathematics

Publications and Presentations

and Statistics

3-28-2013

\title{
Some New Applications of P-P Plots
}

Isha Dewan

Subhash C. Kochar

Portland State University, kochar@pdx.edu

Follow this and additional works at: https://pdxscholar.library.pdx.edu/mth_fac

Part of the Mathematics Commons, and the Statistics and Probability Commons

Let us know how access to this document benefits you.

\section{Citation Details}

Dewan, I. and Kochar, S. (2013). Some new applications of P-P plots. Probability in the Engineering and Informational Sciences, available on CJ02013.

This Article is brought to you for free and open access. It has been accepted for inclusion in Mathematics and Statistics Faculty Publications and Presentations by an authorized administrator of PDXScholar. Please contact us if we can make this document more accessible: pdxscholar@pdx.edu. 


\section{Probability in the Engineering and Informational Sciences \\ http://journals.cambridge.org/PES}

Additional services for Probability in the Engineering and Informational Sciences:

Email alerts: $\underline{\text { Click here }}$

Subscriptions: Click here

Commercial reprints: Click here

Terms of use : $\underline{\text { Click here }}$

\section{SOME NEW APPLICATIONS OF P-P PLOTS}

Isha Dewan and Subhash Kochar

Probability in the Engineering and Informational Sciences / Volume 27 / Issue 03 / July 2013, pp 353 - 366 DOI: 10.1017/S0269964813000077, Published online: 28 March 2013

Link to this article: http://journals.cambridge.org/abstract_S0269964813000077

How to cite this article:

Isha Dewan and Subhash Kochar (2013). SOME NEW APPLICATIONS OF P-P PLOTS.

Probability in the Engineering and Informational Sciences, 27, pp 353-366 doi:10.1017/

S0269964813000077

Request Permissions : $\underline{\text { Click here }}$ 


\title{
SOME NEW APPLICATIONS OF P-P PLOTS
}

\author{
ISHA DEWAN \\ Indian Statistical Institute \\ New Delhi, India \\ E-mail: isha@isid.ac.in \\ SUBHASH KOCHAR \\ Fariborz Maseeh Department of Mathematics and Statistics \\ Portland State University, Portland, OR \\ E-mail: kochar@pdx.edu
}

\begin{abstract}
The P-P plot is a powerful graphical tool to compare stochastically the magnitudes of two random variables. In this note, we introduce a new partial order, called $\mathrm{P}-\mathrm{P}$ order based on $\mathrm{P}-\mathrm{P}$ plots. For a pair of random variables $\left(X_{1}, Y_{1}\right)$ and $\left(X_{2}, Y_{2}\right)$ one can see the relative precedence of $Y_{2}$ over $X_{2}$ versus that of $Y_{1}$ over $X_{1}$ using $\mathrm{P}-\mathrm{P}$ order. We show that several seemingly very technical and difficult concepts like convex transform order and super-additive ordering can be easily explained with the help of this new partial order. Several concepts of positive dependence can also be expressed in terms of $\mathrm{P}-\mathrm{P}$ orders of the conditional distributions.
\end{abstract}

\section{INTRODUCTION}

Consider an experiment in which $k$ treatments are to be compared with their controls and we are interested in identifying the treatment which is most effective. A similar situation can also arise if we want to examine the effectiveness of a treatment relative to its control across different experimental conditions or different patient populations. The classical parametric approach would be to compare the $k$ differences between the means of the treatment and the control effects or their standardized versions if the variances differ. As discussed in Holmgren [8] such an approach may not be appropriate unless the underlying random variables belong to the same parametric family differing only in their location parameters. Here is an illustration of this fact.

Let $X_{1}, \ldots X_{4}$ be independent random variables such that $X_{i} \sim N\left(\mu_{i}, \sigma_{i}^{2}\right), i=1, \ldots, 4$. Then,

$$
P\left[X_{2}>X_{1}\right]=\Phi\left(\frac{\mu_{2}-\mu_{1}}{\sqrt{\left(\sigma_{1}^{2}+\sigma_{2}^{2}\right)}}\right)
$$

and

$$
P\left[X_{4}>X_{3}\right]=\Phi\left(\frac{\mu_{4}-\mu_{3}}{\sqrt{\left(\sigma_{3}^{2}+\sigma_{4}^{2}\right)}}\right) .
$$


It can be seen that $\mu_{4}-\mu_{3}>\mu_{2}-\mu_{1}$ may not imply $P\left[X_{4}>X_{3}\right] \leq P\left[X_{2}>X_{1}\right]$ for certain choices of $\sigma_{i}^{2}$ 's. Thus, it does not always make sense to make such comparisons by looking at only the differences of the treatment means and the control means. The same is true if we make decisions based on differences of medians or percentiles of a certain order. Such comparisons become even less meaningful if the underlying distributions belong to different families.

Let $X$ and $Y$ be two continuous type random variables with cumulative distribution functions $F$ and $G$, survival functions $\bar{F}=1-F$ and $\bar{G}=1-G$ and density functions $f$ and $g$, respectively. We use the notation $F^{-1}$ to denote the right continuous inverse of $F$. For the sake of convenience, let us think of $F$ as the control distribution and $G$ as the treatment distribution. The $\mathrm{P}-\mathrm{P}$ plot is defined as the graph of $G$ versus $F$ and is thus expressed in the function form as $G F^{-1}$. Since the $\mathrm{P}-\mathrm{P}$ plot can be expressed as

$$
G F^{-1}(p)=P\left[Y<F^{-1}(p)\right]=P[F(Y)<p]
$$

the $\mathrm{P}-\mathrm{P}$ plot is the distribution function of $F(Y)$. Since $F($.$) transforms X$ into a random variable with uniform distribution on $[0,1]$, the $\mathrm{P}-\mathrm{P}$ plot is a graph of the treatment distribution with the measurement scale transformed so that the control distribution is uniform on the unit interval.

In this paper, we further study the stochastic properties of $\mathrm{P}-\mathrm{P}$ plots and see how it can help in understanding the various concepts in probability and statistics. Let us first recall some notions of stochastic orders.

- $X$ is said to be smaller than $Y$ according to likelihood ratio ordering, denoted by $X \leq_{\operatorname{lr}} Y$, if $g(x) / f(x)$ is nondecreasing in $x$, where $f$ and $g$ denote the densities of $X$ and $Y$, respectively.

- $X$ is said to be smaller than $Y$ in the hazard rate order, denoted by $X \leq_{\mathrm{hr}} Y$, if

$$
P(X-t>x \mid X>t) \leq P(Y-t>x \mid Y>t), \quad \text { for all } x \geq 0 \text { and all } t .
$$

- $X$ is said to be smaller than $Y$ in the reverse hazard rate order, denoted by $X \leq_{\mathrm{rh}} Y$, if

$$
P(t-X>x \mid X \leq t) \geq P(t-Y>x \mid Y \leq t), \quad \text { for all } x \geq 0 \text { and all } t .
$$

- $X$ is said to be stochastically smaller than $Y$ written as $X \leq_{\text {st }} Y$ if

$$
G(x) \leq F(x), \forall x
$$

We have the following chain of implications among the above stochastic orders

$$
\begin{array}{ccc}
X \leq_{\mathrm{lr}} Y & \Longrightarrow & X \leq_{\mathrm{hr}} Y \\
\Downarrow \\
X \leq_{\mathrm{rh}} Y & \Longrightarrow & \\
\Downarrow & \leq_{\mathrm{st}} Y .
\end{array}
$$

The next theorem shows the usefulness of $\mathrm{P}-\mathrm{P}$ plots as a powerful graphical tool in detecting the above stochastic orders.

\section{TheOREM 1.1:}

(a) $X \leq_{\operatorname{lr}} Y$ if and only if the $P-P$ plot $G F^{-1}$ is convex. 
(b) $X \leq_{\mathrm{hr}} Y$ if and only if the $P-P$ plot $G F^{-1}$ is convex at 1. That is,

$$
G F^{-1}(p+(1-p) u) \leq p+(1-p) G F^{-1}(u) \text { for all } p \in(0,1) \text { and all } u \in[0,1]
$$

assuming that $G F^{-1}(1)=1$.

(c) $X \leq_{\mathrm{rh}} Y$ if and only if the $P-P$ plot $G F^{-1}$ is convex at 0 . That is,

$$
G F^{-1}(p u) \leq p G F^{-1}(u) \text { for all } p \in(0,1) \text { and all } u \in[0,1],
$$

assuming that $G F^{-1}(0)=0$.

(d) $X \leq_{\mathrm{st}} Y$ if and only if the $P-P$ plot $G F^{-1}$ is entirely below the 45 degree line.

\section{PROOF:}

(a) Since

$$
\frac{d}{d x} G F^{-1}(u)=\frac{g F^{-1}(u)}{f F^{-1}(u)}
$$

is nondecreasing in $u$ if $G F^{-1}$ is convex, it follows that $\frac{g(x)}{f(x)}$ is nondecreasing in $x$ in that case, since $F^{-1}(u)$ is a nondecreasing function of $u$.

(b) Let $X_{t} \equiv\{X-t \mid X>t\}$ denote the residual life of $X$ at time $t$. It is known that

$$
\begin{aligned}
X \leq_{\mathrm{hr}} Y & \Leftrightarrow X_{t} \leq_{\mathrm{st}} Y_{t} \text { for all } t \\
& \Leftrightarrow F_{t}^{-1}(p) \leq G_{t}^{-1}(p) \text { for all } p \in[0,1] \text { and for all } t,
\end{aligned}
$$

where $F_{t}$ denotes the distribution function of $X_{t}$. It is easy to see that

$$
F_{t}^{-1}(p)=F^{-1}[1-(1-p) \bar{F}(t)]-t,
$$

where $\bar{F}=1-F$, denotes the survival function of $X$.

Therefore, (1.3) holds if and only if

$$
\begin{aligned}
F^{-1} & {[1-(1-p) \bar{F}(t)] \leq G^{-1}[1-(1-p) \bar{G}(t)], \text { for all } p \in[0,1] \text { and for all } t, } \\
\Leftrightarrow & G F^{-1}[p+(1-p) F(t)] \leq p+(1-p) G(t), \text { for all } p \in[0,1] \text { and for all } t, \\
\Leftrightarrow & G F^{-1}[p \cdot 1+(1-p) u] \leq p G F^{-1}(1)+(1-p) G F^{-1}(u), \\
& \quad \text { for all } p \in[0,1] \text { and for all } u \in[0,1],
\end{aligned}
$$

assuming that $G F^{-1}(1)=1$. This proves the desired result.

(c) The proof is similar to the one in part (b).

(d) The proof is obvious.

Remarks:

(a) The condition $(1.1)$ means that any chord from $(1,1)$ to any point on the P-P plot is entirely above the $\mathrm{P}-\mathrm{P}$ plot.

(b) The condition (1.2) means that any chord from $(0,0)$ to any point on the $\mathrm{P}-\mathrm{P}$ plot is entirely above the $\mathrm{P}-\mathrm{P}$ plot. We call such a function as star-shaped.

In Figure 1, we plot the $\mathrm{P}-\mathrm{P}$ plot of $G$ versus $F$, where $F$ is the distribution function of a parallel system having 3 independent exponential lifetimes each with same failure rate 


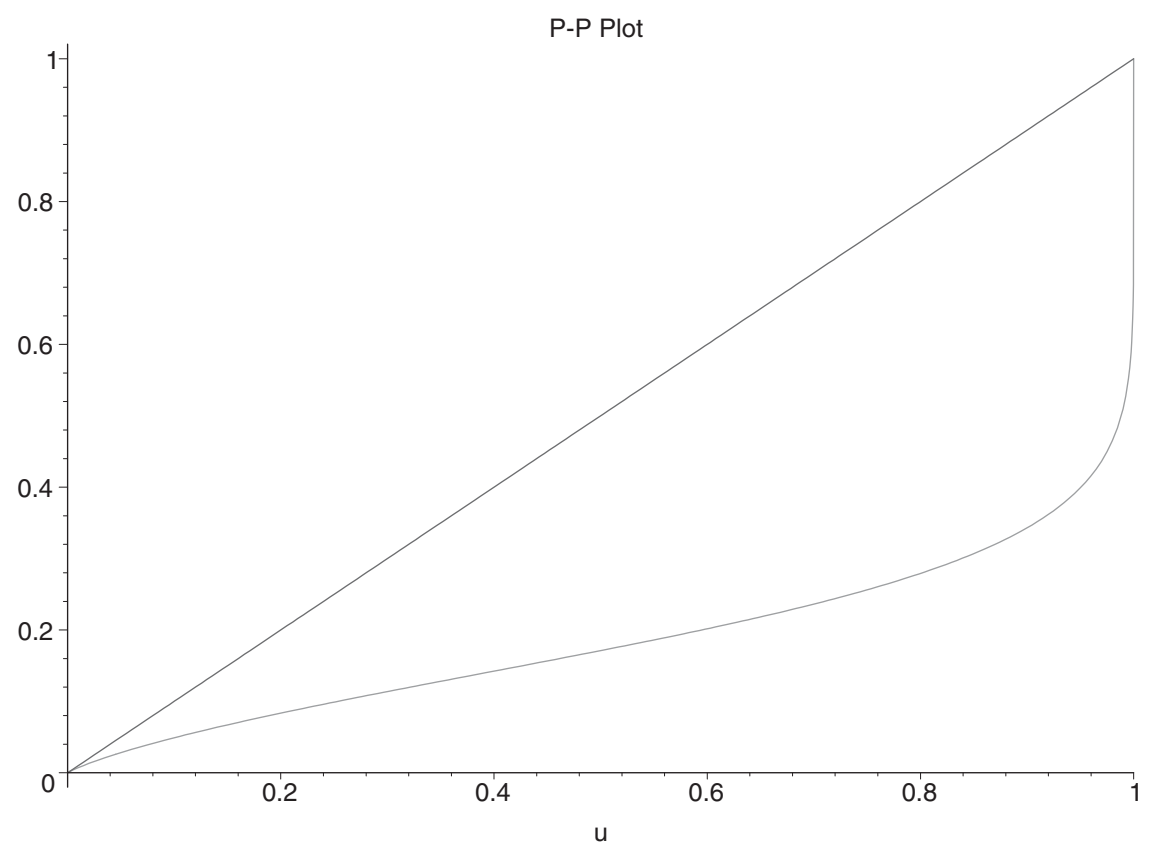

Figure 1. P-P Plot showing stochastic ordering.

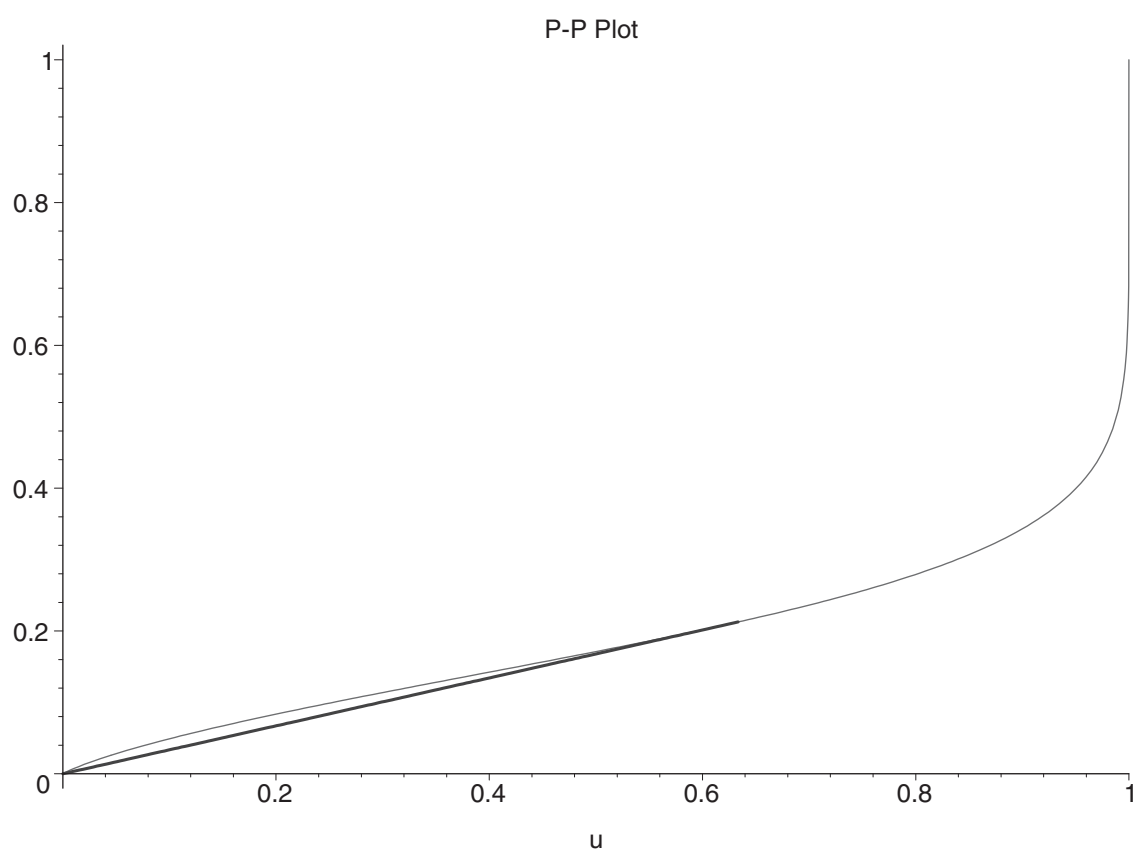

Figure 2. P-P Plot showing stochastic ordering but not RH ordering.

2 and $G$ is the distribution function of a parallel system having 3 independent exponential lifetimes with failure rates $1 / 4,4,8$, respectively. We see that the $\mathrm{P}-\mathrm{P}$ plot lies entirely below the 45 degree line, thus showing that $G$ is stochastically greater than $F$. In Figure 2, we see 


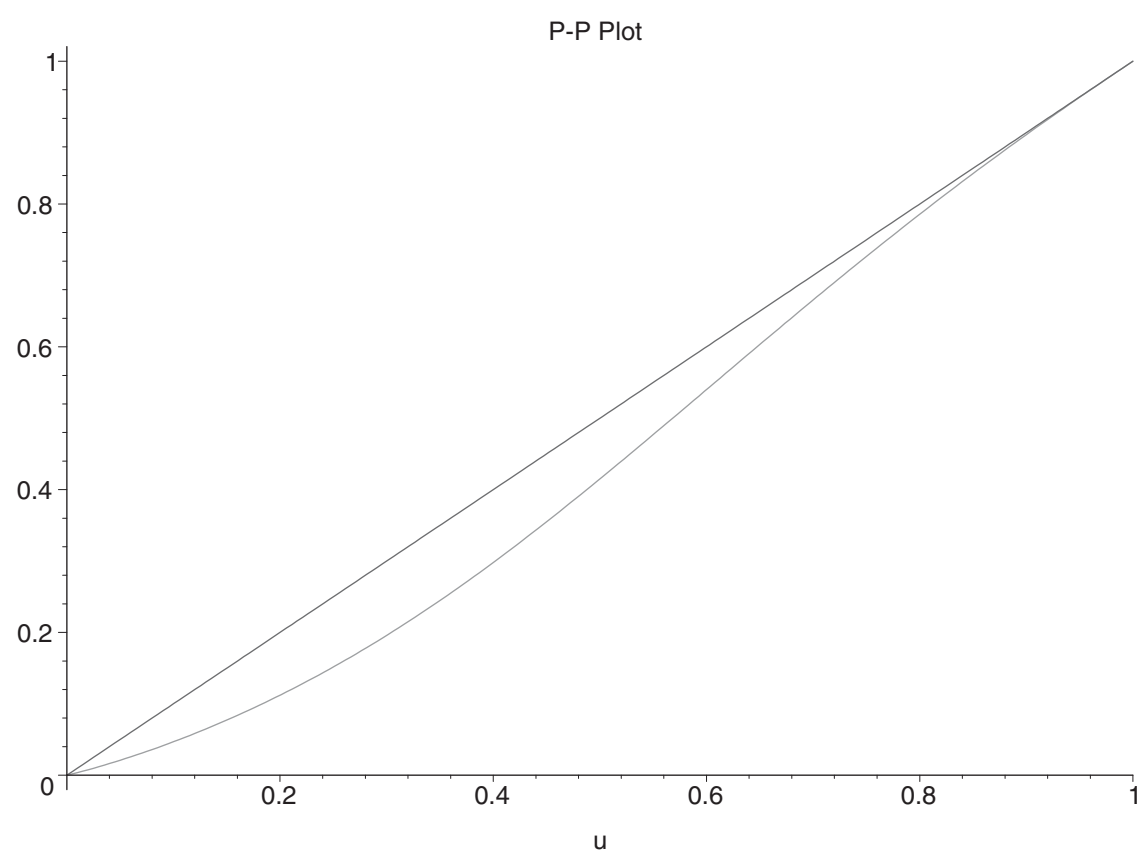

Figure 3. P-P Plot showing stochastic ordering.

that the chord joining $(0,0)$ to the point $\left(0.6335, G F^{-1}(0.6335)\right)$ on this $\mathrm{P}-\mathrm{P}$ plot does not lie above the $\mathrm{P}-\mathrm{P}$ plot. This is an example where $G$ is stochastically greater than $F$, but they are not ordered according to reverse hazard rate ordering. However, any chord from $(1,1)$ to any point on the $\mathrm{P}-\mathrm{P}$ plot lies entirely above the curve confirming that the hazard rate of $G$ is smaller than that of $F$. A theoretical justification of this observation can be found in Khaledi and Kochar [9].

Figure 3 shows the P-P plot of $G$ versus $F$ where $F(x)$ and $G(x)$ are the distribution functions of two-component parallel systems with two independent exponential lifetimes with failure rates $(2,15)$ and $(2,5)$, respectively. Figure 4 shows that a chord from $(1,1)$ to $\left(0, G F^{-1}(0, .45)\right)$ does not lie above the $\mathrm{P}-\mathrm{P}$ plot, indicating that hazard rate ordering does not hold. However, the stochastic ordering holds since the $\mathrm{P}-\mathrm{P}$ plot is entirely below the 45 degree line. In fact, any chord from $(0,0)$ to any point on the $\mathrm{P}-\mathrm{P}$ plot lies above the chord indicating reverse hazard rate ordering between $F$ and $G$.

\subsection{The $\mathbf{P}-\mathbf{P}$ order}

As seen above, $X \leq_{\text {st }} Y$ if and only if the $\mathrm{P}-\mathrm{P}$ plot of $G$ versus $F$ lies entirely below the 45 degree line. The same idea can be extended to compare two random pairs $\left(X_{1}, Y_{1}\right)$ and $\left(X_{2}, Y_{2}\right)$. We want to see whether the increase (or dominance) of $Y_{2}$ over $X_{2}$ is more than that of $Y_{1}$ over $X_{1}$. In the context of examining the efficacy of a Treatment as compared to the Control, we may like to see in which population Treatment is most effective.

Definition 1.2: We say that a pair of random variables $\left(X_{2}, Y_{2}\right)$ is preferred to another pair of random variables $\left(X_{1}, Y_{1}\right)$ according to $P-P$ order (written as $\left(X_{2}, Y_{2}\right) \succ_{\mathrm{PP}}\left(X_{1}, Y_{1}\right)$ ) if and only if the $P-P$ plot of $\left(X_{2}, Y_{2}\right)$ is below that of $\left(X_{1}, Y_{1}\right)$. That is, if and only if,

$$
G_{2} F_{2}^{-1}(u) \leq G_{1} F_{1}^{-1}(u), \quad \forall u \quad \text { in }[0,1] \text {. }
$$




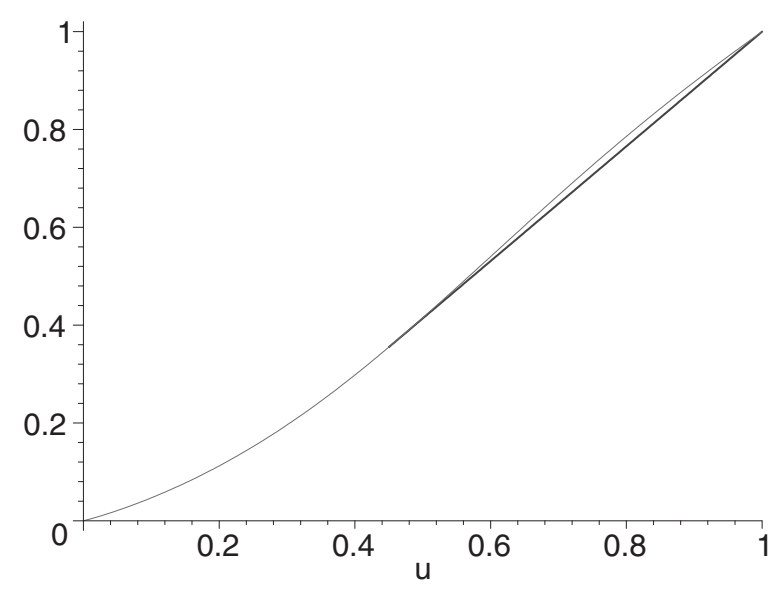

Figure 4. P-P Plot showing stochastic ordering but not HR ordering.

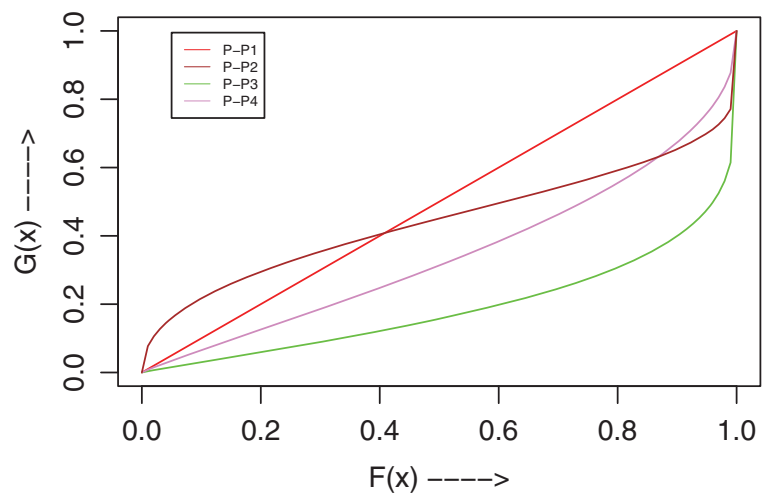

Figure 5. (Color online) P-P plots of Weibull distributions.

Let $F(x)=1-\exp \left(-\left(x / a_{F}\right)^{b_{F}}\right)$ and $G(x)=1-\exp \left(-\left(x / a_{G}\right)^{b_{G}}\right)$ be two Weibull distribution functions. Then

$$
G F^{-1}(p)=1-\exp \left(-a^{b_{G}}[-\log (1-p)]^{b}\right),
$$

where $a=a_{F} / a_{G}$ and $b=b_{G} / b_{F}$.

Figure 5 shows the $\mathrm{P}-\mathrm{P}$ plots of Weibull distributions for various choices of the scale and shape parameters. Let $\left(F_{1}, G_{1}\right),\left(F_{2}, G_{2}\right)$, and $\left(F_{3}, G_{3}\right)$ be three pairs of Weibull distributions with shape parameters $(2,1.818),(0.899,0.818)$, and $(0.899,0.428)$, respectively. The ratio of the scale parameters $a=0.4545$ in all the three cases. Then, clearly $G_{2} F_{2}^{-1}(u) \leq G_{1} F_{1}^{-1}(u)$, (green curve is below the violet curve) clearly indicating that $\left(X_{2}, Y_{2}\right) \succ_{\mathrm{PP}}\left(X_{1}, Y_{1}\right)$. The brown curve crosses the other two curves, showing that this is a partial order. There is no $\mathrm{P}-\mathrm{P}$ order between $\left(X_{1}, Y_{1}\right)$ and $\left(X_{3}, Y_{3}\right)$ and $\left(X_{2}, Y_{2}\right)$ and $\left(X_{3}, Y_{3}\right)$. The red line indicates the $\mathrm{P}-\mathrm{P}$ plot with $F(x)=G(x)$.

For $0 \leq p \leq 1$, let $\xi_{p, 1}$ and $\xi_{p, 2}$ denote the quantiles of order $p$ of $F_{1}$ and $F_{2}$, respectively. Then, $\left(X_{2}, Y_{2}\right) \succ_{\mathrm{PP}}\left(X_{1}, Y_{1}\right)$ means that

$$
G_{2}\left(\xi_{p, 2}\right) \leq G_{1}\left(\xi_{p, 2}\right) \Leftrightarrow P\left[Y_{2} \geq \xi_{p, 2}\right] \geq P\left[Y_{1} \geq \xi_{p, 2}\right] \text { for all } p \in(0,1) \text {. }
$$


As explained in Holmgren [7], with the help of $\mathrm{P}-\mathrm{P}$ plots, treatments and controls from different studies can be compared directly to see the relative magnitudes of different treatment effects as if the control distributions are homogeneous across studies.

Remarks: If in (1.4) above

(a) $F_{1}=G_{1}$, then $G_{2} F_{2}^{-1}(x) \leq x$, that is, $X_{2}<_{\text {st }} Y_{2}$,

(b) $F_{2}=G_{2}$, then $G_{1} F_{1}^{-1}(x) \geq x$, that is, $X_{1}>_{\text {st }} Y_{1}$.

(c) It is easy to show that (1.4) is also equivalent to

$$
F_{2}^{-1} F_{1}(x) \leq G_{2}^{-1} G_{1}(x) \text { for all } x .
$$

Theorem 1.3: Let $X_{i}$ and $Y_{i}$ be independent for $i=1,2$. Then

$$
\left(X_{2}, Y_{2}\right) \succ_{\mathrm{PP}}\left(X_{1}, Y_{1}\right) \Rightarrow P\left(X_{2} \leq Y_{2}\right) \geq P\left(X_{1} \leq Y_{1}\right)
$$

Proof: $G_{2} F_{2}^{-1}(u) \leq G_{1} F_{1}^{-1}(u)$ for all $u \in(0,1)$ implies that

$$
\int_{0}^{1} G_{2} F_{2}^{-1}(u) d u \leq \int_{0}^{1} G_{1} F_{1}^{-1}(u) d u
$$

which is equivalent to

$$
\begin{gathered}
\int_{-\infty}^{\infty} G_{2}(x) d F_{2}(x) \leq \int_{-\infty}^{\infty} G_{1}(x) d F_{1}(x) \\
\Leftrightarrow P\left(Y_{2}<X_{2}\right) \leq P\left(Y_{1}<X_{1}\right) \\
\Leftrightarrow P\left(X_{2} \leq Y_{2}\right) \geq P\left(X_{1} \leq Y_{1}\right) .
\end{gathered}
$$

Now we look at some models which lead to $\mathrm{P}-\mathrm{P}$ ordering between two pairs of independent random variables.

\subsection{The Location Family}

Let $X_{1}$ and $X_{2}$ be two continuous random variables belonging to a location family of distributions and let $Y_{1}$ and $Y_{2}$ belong to possibly another location family of distributions. It is shown in the next theorem that if the differences of location parameters in the two populations are ordered, then the pair of random variables are $\mathrm{P}-\mathrm{P}$ ordered.

TheOREm 1.4: Suppose $F_{1}(x)=F\left(x-\mu_{1}\right), G_{1}(x)=G\left(x-\mu_{2}\right) ; F_{2}(x)=F\left(x-\mu_{3}\right), G_{2}(x)=$ $G\left(x-\mu_{4}\right)$ for every $x$. Further assume that $\mu_{2}-\mu_{1}<\mu_{4}-\mu_{3}$. Then $\left(X_{2}, Y_{2}\right) \succ_{\mathrm{PP}}\left(X_{1}, Y_{1}\right)$.

Proof: It is easy to see that $F_{1}^{-1}(u)=\mu_{1}+F^{-1}(u)$. Then

$$
G_{1} F_{1}^{-1}(u)=G_{1}\left[\mu_{1}+F^{-1}(u)\right]=G\left[\mu_{1}-\mu_{2}+F^{-1}(u)\right] .
$$

Similarly

$$
G_{2} F_{2}^{-1}(u)=G_{2}\left[\mu_{3}+F^{-1}(u)\right]=G\left[\mu_{3}-\mu_{4}+F^{-1}(u)\right] .
$$

Since $\mu_{2}-\mu_{1}<\mu_{4}-\mu_{3}$, it follows that $G_{2} F_{2}^{-1}(u) \leq G_{1} F_{1}^{-1}(u)$ for all $u \in(0,1)$. 


\subsection{The Scale Family}

A similar result holds for the scale family of distributions for non-negative random variables.

TheOREM 1.5: Suppose $F_{1}(x)=F\left(x / \sigma_{1}\right), G_{1}(x)=G\left(x / \sigma_{2}\right) ; F_{2}(x)=F\left(x / \sigma_{3}\right), G_{2}(x)=$ $G\left(x / \sigma_{4}\right)$ for $x \geq 0$. Further assume that $\frac{\sigma_{3}}{\sigma_{4}}<\frac{\sigma_{1}}{\sigma_{2}}$. Then $\left(X_{2}, Y_{2}\right) \succ_{\mathrm{PP}}\left(X_{1}, Y_{1}\right)$.

Proof: It is easy to see that $F_{1}^{-1}(x)=\sigma_{1} F^{-1}(x)$. Then

$$
G_{1} F_{1}^{-1}(x)=G_{1}\left[\sigma_{1} F^{-1}(x)\right]=G\left[\frac{\sigma_{1}}{\sigma_{2}} F^{-1}(x)\right] .
$$

Similarly

$$
G_{2} F_{2}^{-1}(x)=G_{2}\left[\sigma_{3} F^{-1}(x)\right]=G\left[\frac{\sigma_{3}}{\sigma_{4}} F^{-1}(x)\right] .
$$

Since $\sigma_{3} / \sigma_{4}<\sigma_{1} / \sigma_{2}$, the result follows.

In particular, results in Theorems 1.4 and 1.5 hold if all the four random variables belong to the same location and scale family, respectively.

\subsection{The Proportional Hazard Rate Family}

Theorem 1.6: Suppose $\bar{F}_{1}(x)=\bar{F}^{\alpha_{1}}(x), \bar{G}_{1}(x)=\bar{F}^{\alpha_{2}}(x)$ and $\bar{F}_{2}(x)=\bar{G}^{\alpha_{3}}(x), \bar{G}_{2}(x)=$ $\bar{G}^{\alpha_{4}}(x)$. Then $\frac{\alpha_{2}}{\alpha_{1}}>\frac{\alpha_{4}}{\alpha_{3}}$ implies $\left(X_{2}, Y_{2}\right) \succ_{\mathrm{PP}}\left(X_{1}, Y_{1}\right)$.

PROOF: The proof follows once we note that $G_{1} F_{1}^{-1}(u)=1-(1-u)^{\frac{\alpha_{2}}{\alpha_{1}}}$ and $G_{2} F_{2}^{-1}(u)=$ $1-(1-u)^{\frac{\alpha_{4}}{\alpha_{3}}}$ and since $0 \leq(1-u) \leq 1$.

\subsection{The Proportional Reverse Hazard Rate Family}

TheOREM 1.7: Suppose $F_{1}(x)=F^{\alpha_{1}}(x), G_{1}(x)=F^{\alpha_{2}}(x)$ and $F_{2}(x)=G^{\alpha_{3}}(x), G_{2}(x)=$ $G^{\alpha_{4}}(x)$. Then $\frac{\alpha_{2}}{\alpha_{1}}<\frac{\alpha_{4}}{\alpha_{3}}$ implies $\left(X_{2}, Y_{2}\right) \succ_{\mathrm{PP}}\left(X_{1}, Y_{1}\right)$.

Proof: The proof follows once we note that $G_{1} F_{1}^{-1}(u)=u^{\alpha_{2} / \alpha_{1}}$ and $G_{2} F_{2}^{-1}(u)=u^{\alpha_{4} / \alpha_{3}}$ and since $0 \leq u \leq 1$.

In this note we show that several partial orderings and concepts on stochastic orderings can be conveniently interpreted in terms of $\mathrm{P}-\mathrm{P}$ order. In Section 2, we show that various aging orders like convex transform ordering and super-additive ordering can be easily explained by comparing the $\mathrm{P}-\mathrm{P}$ plots of the residual life times. The last section is devoted to the study of various positive dependence orders where we show that these can be expressed in terms of the $\mathrm{P}-\mathrm{P}$ orders between appropriate conditional distributions.

\section{AGING COMPARISONS}

In this section, we show that several aging orderings of life distributions can be easily explained with the help of $\mathrm{P}-\mathrm{P}$ orders between the residual life times. 


\subsection{More IFR Comparisons}

The residual life of a random variable $X$ at time $t$ is given by $X_{t}=X-t \mid X>t$. A random variable $X$ with cdf $F$ is said to have increasing failure rate (IFR) distribution if $X_{t}$ is stochastically decreasing in $t$. That is, if and only if for all $s<t, X_{t} \leq_{\mathrm{st}} X_{s}$ or equivalently if

$$
X_{\xi_{q}} \leq_{\text {st }} X_{\xi_{p}} \text { for all } 0 \leq p<q \leq 1
$$

where $\xi_{p}=F^{-1}(p)$ denotes the $p$ th quantile of $X$.

van Zwet [16] introduced the following concept of convex transform ordering to compare the shapes of two probability distributions.

DeFinition 2.1: $X$ is said to be smaller than $Y$ in the convex transform order, denoted by $X \leq_{c} Y$ if and only if, $G^{-1} F(x)$ is convex in $x$ on the support of $X$.

If $X \leq_{c} Y$, then $Y$ is more skewed than $X$ as explained in van Zwet [16] and Marshall and Olkin [14]. The convex transform order is also known as more IFR order in reliability theory, since when $f$ and $g$ exist, the convexity of $G^{-1} F(x)$ is equivalent to

$$
\frac{f\left(F^{-1}(u)\right)}{g\left(G^{-1}(u)\right)}=\frac{r_{X}\left(F^{-1}(u)\right)}{r_{Y}\left(G^{-1}(u)\right)} \text { being increasing in } u \in[0,1],
$$

where $r_{X}$ and $r_{Y}$ denote the failure (or hazard) rates of $X$ and $Y$, respectively. Thus, $X \leq_{c} Y$ can be interpreted to mean that $X$ ages faster than $Y$ in some sense. This partial order is scale invariant. Note that, neither $F$ nor $G$ need to be IFR for this definition to hold. It can be seen that a random variable $X$ has an IFR distribution if and only if it is convex ordered with respect to exponential distribution, which has a constant failure rate. It is known that if $X_{\alpha_{i}}$ has Gamma distribution with shape parameter $\alpha_{i}, i=1,2$, then $X_{\alpha_{1}} \leq_{c} X_{\alpha_{2}}$ for $\alpha_{2} \leq \alpha_{1}$. Kochar and $\mathrm{Xu}$ [12] used this order to compare two parallel systems with exponential components.

The above definition of more IFR ordering does not explicitly relate to the residual lifetimes of random variables. In the next theorem we show that convex ordering can also be expressed in terms of the more $\mathrm{P}-\mathrm{P}$ ordering of the residual lifetimes and thus giving us a better insight into this seemingly technical concept.

Theorem 2.2: $X \leq_{c} Y$ if and only if for all $0 \leq p<q \leq 1$,

$$
\left(X_{\xi_{q}}, X_{\xi_{p}}\right) \succ \mathrm{PP}\left(Y_{\xi_{q}^{*}}, Y_{\xi_{p}^{*}}\right)
$$

where $\xi_{p}^{*}=G^{-1}(p)$ denotes the $p$ th quantile of $Y$.

Proof: As noted earlier, the survival function of $X_{t}$ is

$$
\bar{F}_{t}(x)=\frac{\bar{F}(t+x)}{\bar{F}(t)} \text { and } F_{t}^{-1}(x)=F^{-1}[1-(1-x) \bar{F}(t)]-t .
$$

Suppose that (2.2) holds. In the light of the equivalence of (1.4) and (1.5), (2.2) is equivalent to

or equivalently

$$
F_{\xi_{q}}^{-1} G_{\xi_{q}^{*}}(x) \leq F_{\xi_{p}}^{-1} G_{\xi_{p}^{*}}(x) \text { for all } x \text { and } 0 \leq p<q \leq 1,
$$

$$
F_{\xi_{p}}^{-1} G_{\xi_{p}^{*}}(x) \text { decreasing in } p \text { for every } x
$$


Now

$$
\begin{aligned}
F_{\xi_{p}}^{-1} G_{\xi_{p}^{*}}(x) & =F^{-1}\left[1-\bar{G}\left(x+\xi_{p}^{*}\right)\right]-\xi_{p} \\
& =F^{-1} G\left(x+G^{-1}(p)\right)-F^{-1}(p)
\end{aligned}
$$

is decreasing in $p$ for every $x$ if and only if

$$
F^{-1} G(x+y)-F^{-1} G(y) \text { is decreasing in } y \text { for every } x \geq 0,
$$

which is equivalent to $F^{-1} G(x)$ being concave or equivalently $G^{-1} F(x)$ being convex. Hence the proof.

The relation expressed by (2.2) gives a natural and intuitive way of comparing the relative aging of two units. As a consequence of Theorem 2.2, it follows that $X \leq_{c} Y$ implies $P\left(X_{\xi_{q}} \leq X_{\xi_{p}}\right) \geq P\left(Y_{\xi_{q}^{*}} \leq Y_{\xi_{p}^{*}}\right)$ for all $0 \leq p<q \leq 1$. If $Y$ has exponential distribution, then the distribution of $Y_{\xi_{p}^{*}}^{*}$ does not depend on $p$ and $P\left(Y_{\xi_{q}^{*}} \leq Y_{\xi_{p}^{*}}\right)=1 / 2$.

\subsection{More New Better Than Used Comparisons}

A random variable $X$ with cdf $F$ is said to be new better than used (NBU) if the residual life of a new component is better than that of a used component which has been working for a time $t$ for all $t>0$. This is equivalent to saying that

$$
X_{t} \leq_{\mathrm{st}} X \text { for all } t>0 .
$$

To compare the relative aging of two arbitrary non-negative random variables $X$ and $Y$ with distribution functions $F$ and $G$, respectively, the following definition of super-additive ordering is well known in the literature (cf. Barlow and Proschan [3]).

Definition 2.3: $X$ is said to be smaller than $Y$ in super-additive order, denoted by $X \leq_{\text {su }}$ $Y$ if and only if, $G^{-1} F(x)$ is super-additive in $x$ on the support of $X$. That is, if and only if

$$
G^{-1} F(x+y) \geq G^{-1} F(x)+G^{-1} F(y) \text { for all } x, y \geq 0 .
$$

If $X \leq_{\text {su }} Y$ holds we also say that $X$ is more $N B U$ than $Y$. Like convex ordering, superadditive ordering is also scale invariant. It is known that convex ordering implies superadditive ordering, but the converse is not true. It is easy to show that if in the above definition, $G$ is exponential, then $F$ is NBU. Again the above definition of super-additive ordering is very technical and does not help in understanding the concept of one distribution aging faster than another. The next theorem gives an easy interpretation of this concept in terms of the $\mathrm{P}-\mathrm{P}$ plots of the residual lifetimes.

Theorem 2.4: $X \leq_{\text {su }} Y$ if and only if for all $0 \leq p \leq 1$,

$$
\left(X_{\xi_{p}}, X\right) \succ_{\mathrm{PP}}\left(Y_{\xi_{p}^{*}}, Y\right)
$$

where $\xi_{p}^{*}=G^{-1}(p)$ denotes the pth quantile of $Y$. 
Proof: Note that (2.6) is equivalent to

$$
\begin{aligned}
F_{\xi_{p}}^{-1} G_{\xi_{p}^{*}}(x) & \leq F^{-1} G(x) \text { for all } x \geq 0 \text { and } p \in(0,1) \\
\Leftrightarrow F^{-1} G\left(x+G^{-1}(p)\right)-F^{-1}(p) & \leq F^{-1} G(x) \text { for all } x \geq 0 \text { and } p \in(0,1) \\
\Leftrightarrow F^{-1} G\left(x+G^{-1}(p)\right) & \leq F^{-1} G(x)+F^{-1}(p) \text { for all } x \geq 0 \text { and } p \in(0,1) \\
\Leftrightarrow F^{-1} G(x+y) & \leq F^{-1} G(x)+F^{-1} G(y) \text { for all } x, y \geq 0 .
\end{aligned}
$$

The last equation is the definition of $F^{-1} G$ being sub-additive or equivalently $G^{-1} F$ being super-additive. Hence the proof.

\subsection{More New Better Than Used in Expectation Comparisons}

Let $\left\{T_{n}, n \geq 1\right\}$ denote the occurrence times of a renewal process with common renewal distribution $F$ and let $X$ be the random variable associated with $F$. Let $A(t)=T_{N(t)+1}-t$ denote the forward recurrence time at time $t$. It is known that

$$
\lim _{t \rightarrow \infty} \frac{1}{t} \int_{t}^{\infty} P[A(s)>x] d s=\frac{1}{\mu} \int_{x}^{\infty} \bar{F}(s) d s .
$$

The distribution with density function $\bar{F}_{e}(x)=[\bar{F}(x)] / \mu$ is called the equilibrium distribution. Let us denote the random variable associated with the equilibrium distribution by $X_{\mathrm{e}}$. If the arrival times $\left\{T_{n}\right\}$ denote the times at which buses arrive at a stand, then $A(t)$ is the amount of time you must wait at the stand for the next bus if you arrive at the stand at time $t$ and $X_{\mathrm{e}}$ is the waiting time for the next bus if you were to arrive at the stand way out in the (infinite) future at random. It is easy to see that $\left[X_{\mathrm{e}}\right]=\frac{E\left[X^{2}\right]}{2 E[X]}$.

Definition 2.5: $X$ is said to be new better than used in expectation (NBUE) if $E[X-$ $t \mid X>t] \leq E[X]$ for all $t \geq 0$.

Kochar and Wiens [11] introduced the concept of more $N B U E$. Let $\mu_{F}(t)=E[X-$ $t \mid X>t]$ denotes the mean residual life function of $X$ at time $t$.

DeFinition 2.6: We say that $F$ is more NBUE than $G$ if

$$
\frac{\mu_{F}\left(F^{-1}(u)\right)}{\mu_{G}\left(G^{-1}(u)\right.} \leq \frac{\mu_{F}}{\mu_{G}} \text { for all } 0<u<1 .
$$

For relation of more $N B U E$ ordering with other partial orders, see Kochar, Li, and Shaked [10].

It is easy to see that $X$ is NBUE if and only if $X_{\mathrm{e}} \leq_{\mathrm{st}} X$. It follows from Proposition 3.1(e) of Kochar and Wiens [11] that $F$ is more $N B U E$ than $G$ if and only if

$$
\left(X_{\mathrm{e}}, X\right) \succ_{\mathrm{PP}}\left(Y_{\mathrm{e}}, Y\right) \text {. }
$$

\section{DEPENDENCE ORDERINGS}

In this section, we use the $\mathrm{P}-\mathrm{P}$ plots of the conditional distributions to describe various dependence orderings. 


\subsection{More Monotone Regression Dependence}

The following definition of monotone regression dependence or stochastic increasingness was introduced by Lehmann [13] and can also be found in Chapter 5 of Barlow and Proschan [3].

DeFinition 3.1: Given two random variables $X$ and $Y$, we say that $Y$ is stochastically increasing in $X$, denoted by $S I(Y \mid X)$, if $P(Y>y \mid X=x)$ is increasing in $x$ for all $y$; or equivalently,

$$
P(Y \leq y \mid X=x) \geq P\left(Y \leq y \mid X=x^{*}\right), \quad x \leq x^{*} .
$$

Observing that when $X$ and $Y$ are continuous, inequality (3.1) can be written as

$$
H_{\left[\xi_{q}\right]} H_{\left[\xi_{p}\right]}^{-1}(u) \leq u \quad \text { for all } 0<u<1 \text { and for } 0<p \leq q<1,
$$

where $\xi_{p}=F^{-1}(p)$ stands for the $p$ th quantile of the marginal distribution of $X$, and $H_{[s]}$ denotes the conditional distribution of $Y$ given $X=s$.

The concept of more regression dependence or more SI was originally considered by Yanagimoto and Okamoto [17] and later extended and further investigated by Schriever [15], Capéraà and Genest [4] and Fang and Joe [6]. The following formulation of more monotone regression dependence as given in Avérous, Genest, and Kochar [2] is copula based.

DeFinition 3.2: $Y_{1}$ is said to be less stochastic increasing (or monotone regression dependent) in $X_{1}$ than $Y_{2}$ is in $X_{2}$, denoted by $\left(Y_{1} \mid X_{1}\right) \preceq_{\mathrm{SI}}\left(Y_{2} \mid X_{2}\right)$, if and only if, for $0 \leq u \leq 1$ and $0<p \leq q<1$,

$$
H_{2,\left[\xi_{2 q}\right]} H_{2,\left[\xi_{2 p}\right]}^{-1}(u) \leq H_{1,\left[\xi_{1 q}\right]} H_{1,\left[\xi_{1 p}\right]}^{-1}(u),
$$

where $\xi_{i p}=F_{i}^{-1}(p)$ stands for the pth quantile of the marginal distribution of $X_{i}$, and $H_{i,[s]}$ denotes the conditional distribution of $Y_{i}$ given $X_{i}=s$, for $i=1,2$.

Let $Y_{i,\left[\xi_{i p}\right]}$ denote the random variable with distribution function $H_{i,\left[\xi_{i p}\right]}$ for $i=1,2$. Then (3.2) is equivalent to

$$
0<p \leq q<1 \Longrightarrow\left(Y_{2,\left[\xi_{2 p}\right]}, Y_{2,\left[\xi_{2 q}\right]}\right) \succ_{\mathrm{PP}}\left(Y_{1,\left[\xi_{1 p}\right]}, Y_{1,\left[\xi_{1 q}\right]}\right)
$$

Avérous et al. [2] used this order to compare the relative degree of dependence among order statistics.

\subsection{More Right Tail Increasing Dependence}

Let $(X, Y)$ be a bivariate random vector with joint cdf $H$ and with marginal cdfs $F$ and $G$, respectively. $Y$ is said to be right tail increasing in $X$ if

$$
x<x^{\prime} \Rightarrow\{Y \mid X>x\} \leq_{\text {st }}\left\{Y \mid X>x^{\prime}\right\},
$$

or equivalently if

$$
0<p \leq q<1 \Rightarrow\left\{Y \mid X>\xi_{p}\right\} \leq_{\text {st }}\left\{Y \mid X>\xi_{q}\right\} .
$$

Let $H_{x}$ denote the cdf of the conditional distribution of $Y$ given $X>x$. Then (3.4) is equivalent to

$$
H_{\xi_{q}} H_{\xi_{p}}^{-1}(u) \leq u \text { for all } 0<u<1 \text { and } 0<p \leq q<1 .
$$

Now for $i=1,2$, let $\left(X_{i}, Y_{i}\right)$ be a pair of continuous random variables with joint cumulative distribution function $H_{i}$ and marginals $F_{i}$ and $G_{i}$. Dolati, Genest, and Kochar [5] 
introduced the following concept of more right tail increasing dependence to compare the relative degree of dependence between two pairs of random variables. This version based on copulas is a modification of the one originally proposed by Avérous and Dortet-Bernadet [1].

DeFinition 3.3: $Y_{2}$ is said to be more right tail increasing in $X_{2}$ than $Y_{1}$ is in $X_{1}$, denoted by $\left(Y_{1} \mid X_{1}\right) \prec_{\mathrm{RTI}}\left(Y_{2} \mid X_{2}\right)$ or $H_{1} \prec_{\mathrm{RTI}} H_{2}$, if and only if

$$
0<p \leq q<1 \Longrightarrow H_{2, \xi_{2 q}} H_{\left.2, \xi_{2 p}\right]}^{-1}(u) \leq H_{1, \xi_{1 q}} H_{1, \xi_{1 p}}^{-1}(u)
$$

for all $u \in(0,1)$, where for $i=1,2, H_{i, s}$ denotes the conditional distribution of $Y_{i}$ given $X_{i}>s$, and $\xi_{i p}=F_{i}^{-1}(p)$ stands for the pth quantile of the marginal distribution of $X_{i}$.

Let $Y_{i, \xi_{i p}}$ denote the random variable with distribution function $H_{i, \xi_{i p}}$ for $i=1,2$. Then (3.6) is equivalent to

$$
0<p \leq q<1 \Longrightarrow\left(Y_{2, \xi_{2 p}}, Y_{2, \xi_{2 q}}\right) \succ_{\mathrm{PP}}\left(Y_{1, \xi_{1 p}}, Y_{1, \xi_{1 q}}\right)
$$

Obviously, (3.6) implies that $Y_{2}$ is right tail increasing in $X_{2}$ if $X_{1}$ and $Y_{1}$ are independent. It also implies that if $Y_{1}$ is stochastically increasing in $X_{1}$, then so is $Y_{2}$ in $X_{2}$; and conversely, if $Y_{2}$ is stochastically decreasing in $X_{2}$, then so is $Y_{1}$ in $X_{1}$. Dolati et al. [5] used this concept to compare the degree of dependence of the largest order statistic on the smallest order statistic in a proportional hazard rate model as a function of the proportionality parameters.

\subsection{More Positive Quadrant Dependence}

Let $(X, Y)$ be a bivariate random vector with joint cdf $H$ and with marginal cdfs $F$ and $G$, respectively. Then the pair $(X, Y)$ is said to be positive quadrant dependent (PQD) (Barlow and Proschan [3]) if

$$
H(x, y) \geq F(x) G(y), \quad \forall x, y
$$

or equivalently if

$$
H\left(\xi_{p}, \xi_{q}\right) \geq p q, \quad \forall 0<p, q<1,
$$

where $F\left(\xi_{p}\right)=p$ and $G\left(\xi_{q}\right)=q$, which can be rewritten as

$$
C(u, v) \geq u v \quad \forall 0<u, v<1,
$$

where $C(u, v)=H\left(\xi_{u}, \xi_{v}\right)$ denotes the copula of the pair $(X, Y)$. Note that $C(u, v)=u v$ if $X$ and $Y$ are independent.

For $i=1,2$, let $\left(X_{i}, Y_{i}\right)$, be a pair of continuous random variables with joint cumulative distribution function $H_{i}$ and marginals $F_{i}$ and $G_{i}$. The following concept of more positive quadrant dependence has been widely discussed in the literature.

Definition 3.4: $Y_{2}$ is said to be more positive quadrant dependent in $X_{2}$ than $Y_{1}$ is in $X_{1}$, denoted by $\left(Y_{1} \mid X_{1}\right) \prec_{\mathrm{PQD}}\left(Y_{2} \mid X_{2}\right)$ or $H_{1} \prec_{\mathrm{PQD}} H_{2}$ if and only if

$$
C_{1}(u, v) \leq C_{2}(u, v) \text { for all } 0 \leq u, v \leq 1,
$$

where $C_{i}$ is the copula of $H_{i}$ for $i=1,2$. 
It is easy to see that (3.9) is equivalent to

$$
0<p<1 \Longrightarrow\left(Y_{2}, Y_{2, \xi_{2 p}}\right) \succ_{\mathrm{PP}}\left(Y_{1}, Y_{1, \xi_{1 p}}\right)
$$

where $Y_{i, \xi_{i p}}, i=1,2$ is as defined in Section 3.2.

We have the following chain of implications among the above partial orderings

$$
\left(Y_{1} \mid X_{1}\right) \preceq_{\mathrm{SI}}\left(Y_{2} \mid X_{2}\right) \Rightarrow\left(Y_{1} \mid X_{1}\right) \preceq_{\mathrm{RTI}}\left(Y_{2} \mid X_{2}\right) \Rightarrow\left(Y_{1} \mid X_{1}\right) \preceq_{\mathrm{PQD}}\left(Y_{2} \mid X_{2}\right) .
$$

\section{Acknowledgments}

Subhash Kochar would like to thank Indian Statistical Institute, New Delhi for their hospitality while on sabbatical leave from Portland State University. This work was partly done during this visit.

\section{References}

1. Avérous, J. \& Dortet-Bernadet, J.L. (2000). LTD and RTI dependence orderings. Canadian Journal of Statististics 28: 151-157.

2. Avérous, J., Genest, C., \& Kochar, S.C. (2005). On the dependence structure of order statistics. Journal of Multivariate Analysis 94: 159-171.

3. Barlow, R.E. \& Proschan, F. (1981). Statistical theory of reliability and life testing. Silver Spring, Maryland: To Begin with.

4. Capera, P. \& Genest C. (1990). Concepts de dependance et orders stochastiques pour des lois bidimensionnelles. Canadian Journal of Statistics 18: 315-326.

5. Dolati, A., Genest C., \& Kochar, S.C. (2008). On the dependence between the extreme order statistics in the proportional hazards model. Journal of Multivariate Analysis 99: 777-786.

6. Fang, Z. \& Joe, H. (1992). Further developments on some dependence orderings for continuous bivariate distributions. Annals of Institute of Statistical Mathematics 44: 501-517.

7. Holmgren, E.B. (1989). Using P-P plots in Meta-analysis as general measures of treatment effects. Technical report - Stanford University.

8. Holmgren, E.B. (1995). The P-P plot as a method for comparing treatment effects. Journal of American Statistical Association 90: 360-365.

9. Khaledi, B. \& Kochar, S.C. (2000). Some new results on stochastic comparisons of parallel systems. Journal of Applied Probability 37: 1123-1128.

10. Kochar, S.C., Li, X., \& Shaked, M. (2002). The total time on test transform and the excess wealth stochastic orders of distributions. Advances in Applied Probability 34(2002), 826-845.

11. Kochar, S.C. \& Weins, D. (1987). Partial orderings of life distributions with respect to their aging properties. Naval Research Logistic 34, 823-829.

12. Kochar, S.C. \& Xu, M. (2009). Comparisons of parallel systems according to convex transform order. Journal of Applied Probability 46: 342-352.

13. Lehmann, E.L. (1966). Some concepts of dependence. Annals of Mathematical Statistiscs 37: $1137-1153$.

14. Marshall, A.W. \& Olkin, I. (2007). Life Distributions, structure of nonparametric, semiparametric, and parametric families. New York: Springer.

15. Schriever, B.F. (1987). An ordering for positive dependence. Annals of Statistics 15: 1208-1214.

16. van Zwet, W.R. (1970). Convex transformations of random variables. Mathematical Centre Tracts No. 7, 2nd ed. Amsterdam: Mathematical Centre.

17. Yanagimoto, T. \& Okamoto, M. (1969). Partial orderings of permutations and monotonicity of a rank correlation statistic. Annals of Institute of Statistical Mathematics 21: 489-506. 